\title{
Rehearsal strategies and the primacy effect in serial learning'
}

\author{
James H. Reynolds and John P. Houston \\ UNIVERSITY OF CALIFORNIA, BERKELEY
}

\begin{abstract}
Abstraet
This study evaluated the effects of three different types of overt rehearsal upon the serial position curve after a single serial learning trial. Ss who rehearsed from the beginning of the list displayed a distinct primacy effect. Rehearsal of successive pairs of items and rehearsal of single items within the list produced minimal and intermediate degrees of primacy, respectively. The curve of a control group, which received no rehearsal instructions, did not differ from that of the group rehearsing from the beginning of the list.
\end{abstract}

\section{Problem}

Previous investigations (Deese, 1957; Krueger, 1932) have indicated the importance of instructions in the determination of serial position effects. The results implied that the instructions determined the strategy of rehearsal employed during learning, and that the shape of the serial curve was dependent.upon the strategy used. The present study explored this implication by evaluating the effects of three specific types of overt serial rehearsal upon the primacy portion of the serial position curve. All Ss received one learning trial and one test trial, but received different instructions in how to rehearse during the learning trial. It was predicted that Ss instructed to rehearse all items in order from the beginning of the list each time a new item was presented (Strategy B) would display a marked primacy effect because beginning items would receive the most practice. Alternatively, Ss instructed to rehearse successive pairs of items, i. e., the current and the preceding item (Strategy P), were expected to yield a flat curve since all items received equal practice. Similarly, repetition of only single items as they appeared for study (Strategy S) was expected to produce a flat curve. The control group, given no rehearsal instructions, was expected to produce the marked primacy effect characteristic of an initial serial learning trial.

\section{Method}

Sixty four University of California undergraduates were randomly assigned to the four conditions, $16 \mathrm{Ss}$ per group. The serial units were 12 common two-syllable high frequency nouns. None had the same first letter and intralist similarity was minimal. Two random orders of the list were developed, and each order was assigned to half of the Ss in each condition. Both the learning and the test trials were conducted at a 4-second rate on a Stowe drum. The various rehearsal instructions were read to the $\mathrm{S}$ before the first, or learning, trial. Instructions for the Strategy B group required the $\mathrm{S}$ to repeat aloud the entire list over and over from the beginning each time a new word appeared on the drum. Ss in the $P$ condition repeated alternately the word in view and the preceding word, thus rehearsing a new pair of items each time a new word was exposed. Strategy S instructions were to practice aloud each word singly as it appeared in the drum window. The control group was told only to learn the words in the order in which they appeared. Typical serial anticipation instructions were given to all groups in the 45-second interval which elapsed between the learning and test trials.

\section{Results and Diseussion}

The mean total correct responses on the test trial for the $B, P, S$, and control conditions were 4.69 $(\mathrm{SEm}=.36), 4.38(\mathrm{SEm}=.78), 2.81(\mathrm{SEm}=.40)$, and $4.56(\mathrm{SEm}=.49)$, respectively. The 12-item list was divided into four three-item blocks representing the beginning, end, and middle list positions, and the mean number of correct responses for each group at each position are plotted in Fig. 1. These block data were used in a $4 \times 4$ repeated measures analysis of variance to evaluate the effects of Position, Rehearsal Strategy, and the Position $x$ Strategy interaction upon test trial performance. The Position effect was highly significant, ( F, 60.71; df, 3/180; $p<.001$,) indicating that serial re-

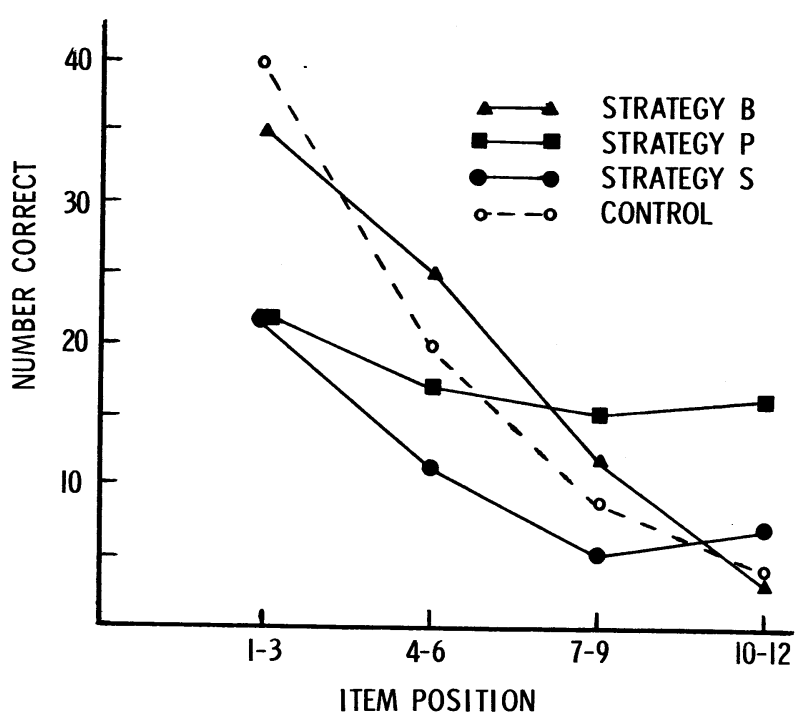

Fig. 1. Correct responses made by the strategy and control groups at each of four serial positions. Each position represents three serial items. 
call was related to list position, as expected. The four strategy conditions did not differ significantly in terms of the total number of correct test trial responses ( $F$, 2.66 ; df, $3 / 60 ; p>.05)$. However, the Position $x$ Rehearsal Strategy interaction was significant, (F, 6.24; df, $9 / 180 ; p<.001$,) indicating reliable differences among groups in the shape of the serial position curve.

A trend analysis of the interaction data indicated that differences among the curves are attributable solely to variations in linear trend, (F, 17.02; $\mathrm{df}, 3 / 180 ; \mathrm{p}<.001$,) with nonlinear trend components failing to reach the .05 level of significance. The slope coefficients of the bestfitting straight lines for the P, S, B, and control groups, obtained by a method described by McNemar (1962, p. $355)$, were $-.06,-.16,-.34$, and -.37 , respectively. The coefficient of nearly zero for Strategy $P$ confirms the prediction that equal rehearsal of all pairs in the list would eliminate the primacy effect from the serial curve. However, a nonorthogonal comparison of the $\mathrm{P}$ and $\mathrm{S}$ strategies indicated that the difference between the slopes of these curves is significant, (F, 3.95; df, 1/135; $\mathrm{p}<.05$, ) implying the existence of a mild primacy effect for Strategy $S$ even though rehearsal of items was distributed evenly over all list positions. Post-experimental interviews, in which $25 \%$ of the Ss in Strategy S but none in the $\mathrm{P}$ or $\mathrm{B}$ groups reported covert repetition of previous items, suggests that this effect may have been due to uncontrolled covert practice occurring in the $S$ condition. Finally, the coefficient obtained for Strategy
B is nearly identical to that of the control group, and reflects a marked primacy effect in both groups as predicted.

The results of this exploratory study support the implication from earlier investigations that the shape of the serial position curve is at least partially dependent upon the strategy of rehearsal employed during learning, and indicate that the distribution of practice over various portions of the list is a critical variable in determining which items will be learned first. The close agreement of the Strategy B and the control group curves suggests that the major rehearsal strategy employed by the $\mathrm{S}$ when first confronted with a serial task is to rehearse repeatedly from the beginning stimulus onward in order of presentation. In contrast, rehearsal of successive pairs of items produces an equivalent amount of learning while virtually eliminating the primacy effect usually found on initial trials of a serial anticipation task.

\section{References}

DEESE, J. Serial organization in the recall of disconnected items. Psychol. Rep., 1957, 3, 577-582.

KRUEGER, W. C. Learning during directed attention. J. exp. Psychol., 1932, 15, 517-527.

McNEMAR, Q. Psychological statistics. (3rd ed.) New York: Wiley, 1962.

\section{Note}

1. This research was conducted during the tenures of the authors' USPHS postdoctoral fellowships at the Institute of Human Learning, University of California, Berkeley.

\begin{abstract}
Abstraet
STEFFY, R. A. (VA Hospital, Danville, Ill.), \& ERIKSEN, C. W. (U. Illinois). Short term perceptual recognition memory for tachistoscopically presented nonsense forms. J. exp. Psychol., in press. -24 male adults were assigned 12 each to 2 conditions. A display consisting of triangular arrangements of 3 Vanderplas and Garvin nonsense forms was tachistoscopically presented. In the center of the trianglar arrangment a 4 th form (cue form) occurred which was identical to 1 of the 3 in the corners. S's task was to indicate which of the 3 forms was identical to the cue form. During experimental trials cue and alternative forms were each exposed for the duration that yielded $90 \%$ accuracy on simultaneous presentations. 2 sequences of presentation were used, 1 where cue form occurred 1st followed at delays
\end{abstract}

of 10-700 ms. by the 3 alternatives, and 2nd, where alternatives were presented 1st. There were 2 conditions; 1 where the adapting and delay fields of the tachisto scope were dark, and the other where they were illuminated. 12 additional Ss were run in an exact replication of the light condition.

For dark adapting and delay fields impairment in recognition was obtained at delays under $100 \mathrm{~ms}$. for both sequences. This was consistent with previously advanced explanation in terms of luminance summation and reduced figure-ground contrast. A marked superiority of the cue-alternative sequence was found in both conditions. This result was shown to be consistent with findings of other studies on short term memory. (Pre-publication copies available from the second author.) 